\title{
FELLER PROPERTY AND INFINITESIMAL GENERATOR OF THE EXPLORATION PROCESS
}

\author{
ROMAIN ABRAHAM AND JEAN-FRANÇOIS DELMAS
}

\begin{abstract}
We consider the exploration process associated to the continuous random tree (CRT) built using a Lévy process with no negative jumps. This process has been studied by Duquesne, Le Gall and Le Jan. This measure-valued Markov process is a useful tool to study CRT as well as super-Brownian motion with general branching mechanism. In this paper we prove this process is Feller, and we compute its infinitesimal generator on exponential functionals and give the corresponding martingale.
\end{abstract}

\section{INTRODUCTION}

The coding of a Lévy continuous random tree (CRT) by its exploration process can be found in Le Gall and Le Jan [7]. They associate to a Lévy process with no negative jumps that does not drift to $+\infty, X=\left(X_{t}, t \geq 0\right)$, a critical or sub-critical continuous state branching process (CSBP) and a Lévy CRT which keeps track of the genealogy of the CSBP. The exploration process $\left(\rho_{t}, t \geq 0\right)$ takes values in the set of finite measures on $\mathbb{R}_{+}$. Informally, for an individual $t \geq 0$, its generation is recorded by its height $H_{t}=\sup \operatorname{Supp} \rho_{t}$, where Supp $\mu$ stand for the closed support of the measure $\mu$ on $\mathbb{R}$. And $\rho_{t}(d v)$ records, for the individual labeled $t$, the "number" of brothers of its ancestor at height $v$ whose labels are larger than $t$.

For instance, the height process $\left(H_{t}, t \geq 0\right)$ of Aldous' CRT [2] is a normalized Brownian excursion, and the exploration process $\left(\rho_{t}, t \geq 0\right)$ is the Lebesgue measure, that is $\rho_{t}$ is the Lebesgue measure on $\left[0, H_{t}\right]$.

The height process, which is enough to code a CRT, is not Markov whereas the exploration process is Markov. The exploration process has been first introduced in order to study superBrownian motion with general branching mechanism (see [4] and [6]). The strong Markov property of the exploration process is a fundamental property and has been proved in [7]. In [1], the exploration process is used in order to construct a fragmentation process associated to a Lévy process using Markov properties and martingale problems.

The goal of this paper is to give some martingales related to the exploration process. We also prove that the exploration process satisfies the Feller property (and hence has càd-làg paths and satisfies the strong Markov property). And we compute the generator of the exploration process for exponential functionals. At this point, let us mention that we will always work with the topology of weak convergence for finite measures. But, as the set of finite measures on $\mathbb{R}_{+}$is not locally compact for this topology, the Feller property does not make sense on that space. So, we must first extend the definition of the transition function

Date: June 7, 2018.

2000 Mathematics Subject Classification. 60J35, 60J80, 60G57.

Key words and phrases. Exploration process, Lévy snake, Feller property, measure valued process, infinitesimal generator.

The research of the second author was partially supported by NSERC Discovery Grants of the Probability group at Univ. of British Columbia. 
of the exploration process to the set of finite measures on $E=\mathbb{R}_{+} \cup\{+\infty\}$ endowed with a metric that makes it compact.

We give the general organization of the paper. In Section 2 we recall the construction and definition of the exploration process and some of its properties (which can be found in [4) that will be used in the sequel. We then state the Feller property, Theorem 2.1. We also give its infinitesimal generator for exponential functionals, Corollary 2.3, as well as the related martingales in Corollaries 2.5 and 2.6. We also recall in Remark 2.8 the relation given in [1] between the infinitesimal generator of the exploration process associated to the Lévy process with Laplace exponent $\psi$ and the infinitesimal generator of the exploration process associated to the Lévy process with Laplace exponent $\psi^{(\theta)}=\psi(\theta+\cdot)-\psi(\theta), \theta \geq 0$. Section 3 is devoted to the proof of the Feller property. We eventually compute the infinitesimal generator of the exploration process for exponential functionals in Section 4

\section{Definitions AND MAIN RESUlts}

2.1. Notations and exploration process. Let $S$ be a metric space, and $\mathcal{B}(S)$ its Borel $\sigma$ field. We denote by $\mathcal{B}_{+}(S)$ (resp. $\left.\mathcal{B}_{b}(S)\right)$ the set of real-valued non-negative (resp. bounded) measurable functions defined on $S$. Let $\mathcal{M}_{f}(S)$ be the set of finite measures defined on $S$ endowed with the topology of weak convergence. We write $\mathcal{M}_{f}$ for $\mathcal{M}_{f}\left(\mathbb{R}_{+}\right)$. For $\mu \in \mathcal{M}_{f}(S)$, $f \in \mathcal{B}_{+}(S)$ we write $\langle\mu, f\rangle$ for $\int_{S} f(x) \mu(d x)$.

We consider a $\mathbb{R}$-valued Lévy process $X=\left(X_{t}, t \geq 0\right)$ with no negative jumps, no Brownian part and starting from 0 . Its law is characterized by its Laplace transform: for $\lambda \geq 0$

$$
\mathbb{E}\left[\mathrm{e}^{-\lambda X_{t}}\right]=\mathrm{e}^{t \psi(\lambda)}
$$

and we suppose that its Laplace exponent, $\psi$, is given by

$$
\psi(\lambda)=\alpha_{0} \lambda+\int_{(0,+\infty)} \pi(d \ell)\left[\mathrm{e}^{-\lambda \ell}-1+\lambda \ell\right]
$$

with $\alpha_{0} \geq 0$ and the Lévy measure $\pi$ is a positive $\sigma$-finite measure on $(0,+\infty)$ such that

$\int_{(0,+\infty)}\left(\ell \wedge \ell^{2}\right) \pi(d \ell)<\infty$ and $\int_{(0,1)} \ell \pi(d \ell)=\infty$. The first assumption (with the condition $\left.\alpha_{0} \geq 0\right)$ implies the process $X$ does not drift to $+\infty$, while the second implies $X$ is a.s. of infinite variation.

For $\mu \in \mathcal{M}_{f}$, we define

$$
H^{\mu}=\sup \operatorname{Supp}(\mu) \in[0, \infty]
$$

where $\operatorname{Supp}(\mu)$ is the closed support of $\mu$, with the convention $\sup \emptyset=0$.

We recall the definition and properties of the exploration process which are given in [7], 6] and 4]. The results of this section are mainly extract from [4].

Let $I=\left(I_{t}, t \geq 0\right)$ be the infimum process of $X: I_{t}=\inf _{0 \leq s \leq t} X_{s}$. We will also consider for every $0 \leq s \leq t$ the infimum of $X$ over $[s, t]$ :

$$
I_{t}^{s}=\inf _{s \leq r \leq t} X_{r}
$$

There exists a sequence $\left(\varepsilon_{n}, n \in \mathbb{N}^{*}\right)$ of positive real numbers decreasing to 0 s.t.

$$
\tilde{H}_{t}=\lim _{k \rightarrow \infty} \frac{1}{\varepsilon_{k}} \int_{0}^{t} \mathbf{1}_{\left\{X_{s}<I_{t}^{s}+\varepsilon_{k}\right\}} d s
$$

exists and is finite a.s. for all $t \geq 0$. 
The point 0 is regular for the Markov process $X-I$, and $-I$ is the local time of $X-I$ at 0 (see 3], chap. VII). Let $\mathbb{N}$ be the associated excursion measure of the process $X-I$ out of 0 , and $\sigma=\inf \left\{t>0 ; X_{t}-I_{t}=0\right\}$ be the length of the excursion of $X-I$ under $\mathbb{N}$. Recall that under $\mathbb{N}, X_{0}=I_{0}=0$.

From Section 1.2 in [4], there exists a $\mathcal{M}_{f}$-valued process, $\rho^{0}=\left(\rho_{t}^{0}, t \geq 0\right)$, called the exploration process, such that :

- For each $t \geq 0$, a.s. $H_{t}^{0}=\tilde{H}_{t}$, where $H_{s}^{0}=H^{\rho_{s}^{0}}$.

- For every $f \in \mathcal{B}_{+}\left(\mathbb{R}_{+}\right)$,

$$
\left\langle\rho_{t}^{0}, f\right\rangle=\int_{[0, t]} d_{s} I_{t}^{s} f\left(H_{s}^{0}\right)
$$

or equivalently

$$
\rho_{t}^{0}(d r)=\sum_{\substack{0<s \leq t \\ X_{s-}<I_{t}^{s}}}\left(I_{t}^{s}-X_{s-}\right) \delta_{H_{s}^{0}}(d r) .
$$

- Almost surely, for every $t>0$, we have $\left\langle\rho_{t}^{0}, 1\right\rangle=X_{t}-I_{t}$.

2.2. The Feller property. In Proposition 1.2.3 4], Duquesne and Le Gall proved that the exploration process is a strong Markov process with càd-làg paths in $\mathcal{M}_{f}$ equipped with the topology of weak convergence (in fact they prove the exploration process is a.s. càd-làg with the variation distance on finite measures). Here, we improve this result by proving that the exploration process fulfills the Feller property.

We set $E=\mathbb{R}_{+} \cup\{+\infty\}$ equipped with a distance that makes $E$ compact. The set $\mathcal{M}_{f}(E)$ is locally compact.

In the definition of the exploration process, as $X$ starts from 0 , we have $\rho_{0}=0$ a.s. To get the Markov property of $\rho$, we must define the process $\rho$ started at any initial measure $\mu \in \mathcal{M}_{f}(E)$.

Let $\mu \in \mathcal{M}_{f}(E)$. For $a \in[0,\langle\mu, 1\rangle]$, we define the erased measure $k_{a} \mu$ by

$$
k_{a} \mu([0, r])=\mu([0, r]) \wedge(\langle\mu, 1\rangle-a), \quad \text { for } r \in E .
$$

If $a>\langle\mu, 1\rangle$, we set $k_{a} \mu=0$. In other words, the measure $k_{a} \mu$ is the measure $\mu$ erased by a mass a backward from $H^{\mu}$, with the natural extension of (11) to $\mathcal{M}_{f}(E)$.

For $\nu, \mu \in \mathcal{M}_{f}(E)$, we define the concatenation $[\mu, \nu] \in \mathcal{M}_{f}(E)$ of the two measures, using the convention $x+\infty=+\infty$ for $x \in E$, by

$$
\langle[\mu, \nu], f\rangle=\langle\mu, f\rangle+\left\langle\nu, f\left(H^{\mu}+\cdot\right)\right\rangle, \quad f \in \mathcal{B}_{+}(E) .
$$

Eventually, we set for every $\mu \in \mathcal{M}_{f}(E)$ and every $t>0$,

$$
\rho_{t}=\left[k_{-I_{t}} \mu, \rho_{t}^{0}\right] \text {. }
$$

We say that $\rho=\left(\rho_{t}, t \geq 0\right)$ is the exploration process started at $\rho_{0}=\mu$, and write $\mathbb{P}_{\mu}$ for its law. We set $H_{t}=H^{\rho_{t}}$. The process $\rho$ is an homogeneous Markov process (this is a direct consequence of Proposition 1.2.3 in [4]).

Theorem 2.1. The exploration process, $\rho$, defined on $\mathcal{M}_{f}(E)$ equipped with the topology of weak convergence is Feller.

The proof of this theorem is given in Section 3. Since $\rho$ has the Feller property we recover it enjoys the strong Markov property and has a.s. càd-làg paths. Notice that if $\mu \in \mathcal{M}_{f}$, as $\rho^{0}$ is $\mathcal{M}_{f}$ valued, we get by construction that $\rho$ is $\mathcal{M}_{f}$ valued. We recover that the exploration 
process in $\mathcal{M}_{f}$ enjoys the strong Markov property and has a.s. càd-làg paths. We can consider the infinitesimal generator of $\rho$.

2.3. The infinitesimal generator. Let $f$ a bounded non-negative function defined on $\mathbb{R}_{+}$ of class $\mathcal{C}^{1}$ with bounded first derivative such that $f(\infty)=\lim _{t \rightarrow \infty} f(t)$ exists.

We consider $F, K \in \mathcal{B}\left(\mathcal{M}_{f}(E)\right)$ defined by: for $\mu \in \mathcal{M}_{f}(E)$,

$$
F(\mu)=\mathrm{e}^{-\langle\mu, f\rangle}, \quad \text { and } \quad K(\mu)=F(\mu)\left[\psi\left(f\left(H^{\mu}\right)\right)-f^{\prime}\left(H^{\mu}\right) \mathbf{1}_{\left\{H^{\mu}<\infty\right\}}\right] .
$$

Notice that $F$ is a continuous function, whereas $K$ belongs only to $\mathcal{B}\left(\mathcal{M}_{f}(E)\right)$ a priori.

We denote by $P_{t}$ the transition function of the exploration process $\rho$ at time $t$ and we consider its resolvent: for $\lambda>0, U_{\lambda}=\int_{0}^{\infty} \mathrm{e}^{-\lambda t} P_{t}$.

Theorem 2.2. Let $\lambda>0$. We have

$$
U_{\lambda}(\lambda F-K)(\mu)=\mathrm{e}^{-\langle\mu, f\rangle}-\frac{f(0)}{\psi^{-1}(\lambda)} \mathrm{e}^{-\psi^{-1}(\lambda)\langle\mu, 1\rangle} .
$$

The proof of this Theorem is given in Section 4 . There exists a local time at $\mu=0$ which has to be considered when computing the infinitesimal generator. This translates into the condition $f(0)=0$ for the domain of the infinitesimal generator, as stated in the next Corollary.

Corollary 2.3. Assume that $f(0)=0, \lambda>0$. We have $U_{\lambda}(\lambda F-K)=F$. In particular $(F, K)$ belongs to the extended domain of the infinitesimal generator of $\rho$.

Remark 2.4. Notice the function $K$ is not continuous. However, it can be proved directly that if $\mu \neq 0$ with compact support then

$$
\lim _{\lambda \rightarrow \infty} \lambda\left(\lambda U_{\lambda} F-F\right)(\mu)=K(\mu)
$$

and if $\mu=0$

$$
\lim _{\lambda \rightarrow \infty} \lambda\left(\lambda U_{\lambda} F-F+\frac{f(0)}{\psi^{-1}(\lambda)}\right)(0)=\psi(f(0))-f^{\prime}(0) .
$$

Standard results on Markov process implies the following Corollary, see e.g. [5] Chapter 4.

Corollary 2.5. Assume $f(0)=0, \lambda \geq 0$. The process $\left(M_{t}, t \geq 0\right)$ defined by

$$
M_{t}=\mathrm{e}^{-\lambda t-\left\langle\rho_{t}, f\right\rangle}+\int_{0}^{t} \mathrm{e}^{-\lambda s-\left\langle\rho_{s}, f\right\rangle}\left[\lambda-\psi\left(f\left(H_{s}\right)\right)+f^{\prime}\left(H_{s}\right) \mathbf{1}_{\left\{H_{s}<\infty\right\}}\right] d s,
$$

where $H_{s}=H^{\rho_{s}}$, is a martingale w.r.t. the filtration generated by $\rho$.

We deduce from the optional stopping Theorem and the monotone class Theorem the next result. (Notice we don't assume $f(0)=0$.)

Corollary 2.6. The process $\left(M_{t}, t \geq 0\right)$ defined by

$$
M_{t}=\mathrm{e}^{-\left\langle\rho_{t \wedge \sigma}, f\right\rangle}-\int_{0}^{t \wedge \sigma} \mathrm{e}^{-\left\langle\rho_{s}, f\right\rangle}\left[\psi\left(f\left(H_{s}\right)\right)-f^{\prime}\left(H_{s}\right) \mathbf{1}_{\left\{H_{s}<\infty\right\}}\right] d s
$$

is a martingale w.r.t. the filtration generated by $\rho$.

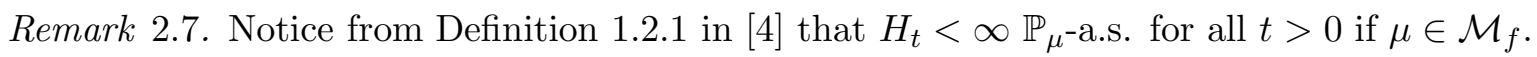
Hence the indicator $\mathbf{1}_{\left\{H_{s}<\infty\right\}}$ can be removed in Corollaries 2.5] and 2.6] under $\mathbb{P}_{\mu}$ for $\mu \in \mathcal{M}_{f}$. 
Remark 2.8. From [1] (see Theorem 6.1 and Proposition 6.3 in Section 6.1), there is a relationship between the infinitesimal generator of $\rho$ and the infinitesimal generator of the exploration process, $\rho^{(\theta)}$, associated to a Lévy process with exponent $\psi^{(\theta)}$, where

$$
\psi^{(\theta)}(\lambda)=\psi(\lambda+\theta)-\psi(\theta), \quad \lambda \geq 0 .
$$

More precisely, let $F, K \in \mathcal{B}\left(\mathcal{M}_{f}\right)$ bounded such that we have $\mathbb{E}_{\mu}\left[\int_{0}^{\sigma}\left|K\left(\rho_{s}\right)\right| d s\right]<\infty$ for any $\mu \in \mathcal{M}_{f}$ and $M_{t}=F\left(\rho_{t \wedge \sigma}\right)-\int_{0}^{t \wedge \sigma} K\left(\rho_{s}\right)$, for $t \geq 0$, defines a martingale under $\mathbb{P}_{\mu}$. Then, we have for all $\mu \in \mathcal{M}_{f}, \mathbb{P}_{\mu}$-a.s.

$$
\int_{0}^{\sigma^{(\theta)}} d u \int_{(0, \infty)}\left(1-\mathrm{e}^{-\theta \ell}\right) \pi(d \ell)\left|F\left(\left[\rho_{u}^{(\theta)}, \ell \delta_{0}\right]\right)-F\left(\rho_{u}^{(\theta)}\right)\right|<\infty
$$

where $\sigma^{(\theta)}=\inf \left\{t>0 ; \rho_{t}^{(\theta)}=0\right\}$, and the process $\left(N_{t}, t \geq 0\right)$ is a martingale, where for $t \geq 0$, $N_{t}=F\left(\rho_{t \wedge \sigma^{(\theta)}}^{(\theta)}\right)-\int_{0}^{t \wedge \sigma^{(\theta)}} d u\left(K\left(\rho_{u}^{(\theta)}\right)+\int_{(0, \infty)}\left(1-\mathrm{e}^{-\theta \ell}\right) \pi(d \ell)\left(F\left(\left[\rho_{u}^{(\theta)}, \ell \delta_{0}\right]\right)-F\left(\rho_{u}^{(\theta)}\right)\right)\right)$.

Now, if we take $F$ and $K$ defined by (3), and assume that $f \geq \varepsilon$ for some constant $\varepsilon>0$. Then, we have

$$
\mathbb{E}_{\mu}\left[\int_{0}^{\sigma}\left|K\left(\rho_{s}\right)\right| d s\right] \leq \mathbb{E}_{\mu}\left[\int_{0}^{\sigma} \mathrm{e}^{-\varepsilon\left\langle\rho_{s}, 1\right\rangle} d s\right]=\frac{1-\mathrm{e}^{-\varepsilon\langle\mu, 1\rangle}}{\psi(\varepsilon)}<\infty .
$$

Notice that $F\left(\left[\mu, \ell \delta_{0}\right]\right)-F(\mu) \leq 0$ and

$$
\begin{aligned}
\int_{(0, \infty)}\left(1-\mathrm{e}^{-\theta \ell}\right) \pi(d \ell)\left(F\left(\left[\mu, \ell \delta_{0}\right]\right)-F(\mu)\right) & =-F(\mu) \int_{(0, \infty)}\left(1-\mathrm{e}^{-\theta \ell}\right)\left(1-\mathrm{e}^{-\ell f\left(H^{\mu}\right)}\right) \pi(d \ell) \\
& =-F(\mu)\left(\psi\left(f\left(H^{\mu}\right)\right)-\psi^{(\theta)}\left(f\left(H^{\mu}\right)\right)\right) .
\end{aligned}
$$

Condition (44) is also satisfied. We get for the martingale $\left(N_{t}, t \geq 0\right)$ given above that

$$
\begin{aligned}
N_{t} & =F\left(\rho_{t \wedge \sigma^{(\theta)}}^{(\theta)}\right)-\int_{0}^{t \wedge \sigma^{(\theta)}} d u\left(K\left(\rho_{u}^{(\theta)}\right)+\int_{(0, \infty)}\left(1-\mathrm{e}^{-\theta \ell}\right) \pi(d \ell)\left(F\left(\left[\rho_{u}^{(\theta)}, \ell \delta_{0}\right]\right)-F\left(\rho_{u}^{(\theta)}\right)\right)\right) \\
& =F\left(\rho_{t \wedge \sigma^{(\theta)}}^{(\theta)}\right)-\int_{0}^{t \wedge \sigma^{(\theta)}} d u F\left(\rho_{s}\right)\left[\psi^{(\theta)}\left(f\left(H^{\rho_{s}^{(\theta)}}\right)\right)-f^{\prime}\left(H^{\rho_{s}^{(\theta)}}\right)\right] .
\end{aligned}
$$

And thanks to Remark 2.7] we recover Corollary 2.6] for the process $\rho^{(\theta)}$ for any initial measure in $\mathcal{M}_{f}$.

\section{Proof of Theorem 2.1}

In this sequel $x_{+}=\max (x, 0)$ denotes the positive part of $x \in \mathbb{R}$.

3.1. A distance which induces the topology of weak convergence. Let $G$ be an increasing non-negative bounded function of class $\mathcal{C}^{1}$ on $\mathbb{R}_{+}$. For convenience, we write $G(\infty)$ for the limit of $G(t)$ as $t$ goes to infinity, and we shall assume that $G(0)=0$ and $G(\infty) \leq 1$. The set $E=\mathbb{R}_{+} \cup\{\infty\}$ endowed with the distance $d(x, y)=|G(x)-G(y)|$ is compact. Then the set of finite measures on $E, \mathcal{M}_{f}(E)$, equipped with the topology of weak convergence is locally compact.

For $r \in \mathbb{R}_{+}, \mu \in \mathcal{M}_{f}(E)$, we set $H_{r}^{\mu}=H^{k_{r} \mu}$. Notice the function $r \mapsto H_{r}^{\mu}$ is non-increasing, right continuous, satisfies $H_{\langle\mu, 1\rangle}^{\mu}=0$. For $r \in[0,\langle\mu, 1\rangle]$, we have $H_{r}^{\mu}=g\left((\langle\mu, 1\rangle-r)^{-}\right)$, where 
$g$ is the right continuous inverse of the cumulative distribution function of $\mu$. In particular, if for $\mu, \nu \in \mathcal{M}_{f}(E)$ we have $\langle\mu, 1\rangle=\langle\nu, 1\rangle$ and $H_{r}^{\mu}=H_{r}^{\nu}$ for all $r \geq 0$, then $\mu=\nu$.

Lemma 3.1. For all $r, v \in \mathbb{R}_{+}, \mu \in \mathcal{M}_{f}(E)$, we have

$$
v<H_{r}^{\mu} \Longleftrightarrow \mu((v,+\infty])>r
$$

For any $h \in \mathcal{B}_{b}(E)$, we have

$$
\int_{0}^{\langle\mu, 1\rangle} h\left(H_{r}^{\mu}\right) d r=\langle\mu, h\rangle
$$

and if $h(0)=0$ this can be also written as

$$
\int_{0}^{\infty} h\left(H_{r}^{\mu}\right) d r=\langle\mu, h\rangle
$$

Proof. Notice that $H_{r}^{\mu}=\sup \{x \in E ; \mu([x,+\infty])>r\}$. This implies (5). Let $h$ be a nonnegative non-decreasing bounded function defined on $\mathbb{R}_{+}$of class $\mathcal{C}^{1}$ such that $h(0)=0$ and $\int_{\mathbb{R}_{+}}\left|h^{\prime}(v)\right| \mu([v,+\infty]) d v$ is finite. We have

$$
\begin{aligned}
\int_{0}^{\infty} h\left(H_{r}^{\mu}\right) d r & =\int_{\mathbb{R}_{+}^{2}} h^{\prime}(v) \mathbf{1}_{\left\{v<H_{r}^{\mu}\right\}} d r d v \\
& =\int_{\mathbb{R}_{+}^{2}} h^{\prime}(v) \mathbf{1}_{\{r<\mu((v,+\infty])\}} d r d v \\
& =\int_{0}^{\infty} h^{\prime}(v) \mu((v,+\infty]) d v \\
& =\int_{\mathbb{R}_{+} \times E} h^{\prime}(v) \mathbf{1}_{\{u>v\}} d v \mu(d u) \\
& =\langle\mu, h\rangle,
\end{aligned}
$$

where we used (5) for the second equality, and $h(0)=0$ for the first and last equality. By linearity and monotone class Theorem, we get (17) for any $h \in \mathcal{B}_{b}(E)$, such that $h(0)=0$. Then (6) is a direct consequence of (7) as $H_{r}^{\mu}=0$ for $r>\langle\mu, 1\rangle$.

In particular, (77) holds for $h=G$. Thus we have $\int_{0}^{\infty} G\left(H_{r}^{\mu}\right) d r \leq\langle\mu, 1\rangle<\infty$ for all $\mu \in \mathcal{M}_{f}(E)$. We introduce the following function defined on $\mathcal{M}_{f}(E)^{2}$ : for $\mu, \nu \in \mathcal{M}_{f}(E)$

$$
\begin{aligned}
D(\mu, \nu) & =|\langle\mu, 1\rangle-\langle\nu, 1\rangle|+\int_{0}^{\infty} d\left(H_{r}^{\mu}, H_{r}^{\nu}\right) d r \\
& =|\langle\mu, 1\rangle-\langle\nu, 1\rangle|+\int_{0}^{\max (\langle\mu, 1\rangle,\langle\nu, 1\rangle)} d\left(H_{r}^{\mu}, H_{r}^{\nu}\right) d r .
\end{aligned}
$$

Since $G$ is bounded by 1 , we have

$$
\langle\mu, 1\rangle \leq D(0, \mu) \leq 2\langle\mu, 1\rangle .
$$

From what precedes Lemma 3.1, we get that $D(\mu, \nu)=0$ implies $\mu=\nu$. It then is easy to check that $D$ is a distance on $\mathcal{M}_{f}(E)$.

Remark 3.2. We deduce from (8) that a sequence $\left(\mu_{n}, n \geq 1\right)$ is unbounded in $\left(\mathcal{M}_{f}(E), D\right)$ if and only if the sequence $\left(\left\langle\mu_{n}, 1\right\rangle, n \geq 1\right)$ is unbounded. 
Lemma 3.3. The topology induced by $D$ on $\mathcal{M}_{f}(E)$ corresponds to the topology induced by the weak convergence.

Notice that $\left(\mathcal{M}_{f}(E), D\right)$ is a locally compact Polish space.

Proof. We first consider a sequence $\left(\mu_{n}, n \geq 1\right)$ of elements of $\mathcal{M}_{f}(E)$ which converges for the distance $D$ to $\mu$. We shall prove that this sequence converges weakly to $\mu$. Let $f \in \mathcal{B}_{b}(E)$ such that $|f(x)-f(y)| \leq d(x, y)$ for all $x, y \in E$. We set $f^{*}$ for $f-f(0)$. Thanks to (7), we have

$$
\begin{aligned}
\left|\left\langle\mu_{n}, f\right\rangle-\langle\mu, f\rangle\right| & =\left|\left\langle\mu_{n}, f(0)\right\rangle-\langle\mu, f(0)\rangle+\int_{0}^{\infty}\left[f^{*}\left(H_{r}^{\mu_{n}}\right)-f^{*}\left(H_{r}^{\mu}\right)\right] d r\right| \\
& \leq|f(0)|\left|\left\langle\mu_{n}, 1\right\rangle-\langle\mu, 1\rangle\right|+\int_{0}^{\infty}\left|f^{*}\left(H_{r}^{\mu_{n}}\right)-f^{*}\left(H_{r}^{\mu}\right)\right| d r \\
& \leq|f(0)|\left|\left\langle\mu_{n}, 1\right\rangle-\langle\mu, 1\rangle\right|+\int_{0}^{\infty} d\left(H_{r}^{\mu_{n}}, H_{r}^{\mu}\right) d r \\
& \leq(|f(0)|+1) D\left(\mu_{n}, \mu\right) .
\end{aligned}
$$

As this holds for any Lipschitz function, we get that $\left(\mu_{n}, n \geq 1\right)$ converges weakly to $\mu$.

Let $\left(\mu_{n}, n \geq 1\right)$ be a sequence of elements of $\mathcal{M}_{f}(E)$ which converges weakly to $\mu$. In particular, the sequence $\left(\left\langle\mu_{n}, 1\right\rangle, n \geq 1\right)$ converges to $\langle\mu, 1\rangle$. Thus, there exists a finite constant $A$ such that $\langle\mu, 1\rangle \leq A$ and $\left\langle\mu_{n}, 1\right\rangle \leq A$ for all $n \geq 1$. Then, we have

$$
\begin{aligned}
\int_{0}^{\infty} d\left(H_{r}^{\mu_{n}}, H_{r}^{\mu}\right) d r & =\int_{0}^{\infty} d r\left|G\left(H_{r}^{\mu_{n}}\right)-G\left(H_{r}^{\mu}\right)\right| \\
& =\int_{0}^{\infty} d r \mid \int_{0}^{\infty} d v G^{\prime}(v)\left[\mathbf{1}_{\left\{r<\mu_{n}((v, \infty])\right\}}-\mathbf{1}_{\{r<\mu((v, \infty])\}}\right] \\
& \leq \int_{\mathbb{R}_{+}^{2}} d r d v G^{\prime}(v)\left|\mathbf{1}_{\left\{r<\mu_{n}((v, \infty])\right\}}-\mathbf{1}_{\{r<\mu((v, \infty])\}}\right| \\
& =\int_{\mathbb{R}_{+}} d v G^{\prime}(v)\left|\mu_{n}((v, \infty])-\mu((v, \infty])\right|,
\end{aligned}
$$

where we used (5) for the second equality. The weak convergence of $\left(\mu_{n}, n \geq 1\right)$ towards $\mu$ implies that $d v$-a.e., $\lim _{n \rightarrow \infty} \mu_{n}((v, \infty])=\mu((v, \infty])$. As $G^{\prime}(v)\left|\mu_{n}((v, \infty])-\mu((v, \infty])\right| \leq$ $2 A G^{\prime}(v)$ and $2 A G^{\prime}$ is integrable, we deduce from dominated convergence Theorem that $\lim _{n \rightarrow \infty} \int_{0}^{\infty} d\left(H_{r}^{\mu_{n}}, H_{r}^{\mu}\right) d r=0$. This and the convergence of $\left(\left\langle\mu_{n}, 1\right\rangle, n \geq 1\right)$ to $\langle\mu, 1\rangle$ imply that $\lim _{n \rightarrow \infty} D\left(\mu_{n}, \mu\right)=0$.

We introduce the distance $D$ in order to get good continuity properties for the concatenation and the erasing functions $k_{a}$.

Lemma 3.4. Let $a \geq 0$. We have $D\left(k_{a} \mu, k_{a} \nu\right) \leq D(\mu, \nu)$.

Proof. Notice $H_{r}^{k_{a} \rho}=H_{r+a}^{\rho}$. We have

$$
\begin{aligned}
D\left(k_{a} \mu, k_{a} \nu\right) & =\left|(\langle\mu, 1\rangle-a)_{+}-(\langle\nu, 1\rangle-a)_{+}\right|+\int_{0}^{\infty} d\left(H_{r+a}^{\mu}, H_{r+a}^{\nu}\right) d r \\
& =\left|(\langle\mu, 1\rangle-a)_{+}-(\langle\nu, 1\rangle-a)_{+}\right|+\int_{a}^{\infty} d\left(H_{r}^{\mu}, H_{r}^{\nu}\right) d r \\
& \leq D(\mu, \nu),
\end{aligned}
$$


where we used for the last inequality the fact that the function $x \mapsto(x-a)_{+}$is Lipschitz with constant equal to one.

With the convention $x+\infty=\infty$ for all $x \in E$, we have

$$
H_{r}^{\left[k_{a} \mu, \rho\right]}= \begin{cases}H_{a}^{\mu}+H_{r}^{\rho} & \text { if } r \leq\langle\rho, 1\rangle, \\ H_{a+r-\langle\rho, 1\rangle}^{\mu} & \text { if } r \geq\langle\rho, 1\rangle .\end{cases}
$$

Let $\mu \in \mathcal{M}_{f}(E)$, and $\mathcal{A}_{\mu} \subset \mathbb{R}_{+}$be the set of continuity points of the function $r \mapsto H_{r}^{\mu}$. Notice that $\mathbb{R}_{+} \backslash \mathcal{A}_{\mu}$ is at most countable.

Lemma 3.5. Let $\left(\mu_{n}, n \geq 1\right)$ be a sequence of $\mathcal{M}_{f}(E)$ which converges weakly to $\mu \in \mathcal{M}_{f}(E)$. For all $a \in \mathcal{A}_{\mu}, \rho \in \mathcal{M}_{f}(E)$, we have that $\left(\left[k_{a} \mu_{n}, \rho\right], n \geq 1\right)$ converges weakly to $\left[k_{a} \mu, \rho\right]$ :

$$
\lim _{n \rightarrow \infty} D\left(\left[k_{a} \mu_{n}, \rho\right],\left[k_{a} \mu, \rho\right]\right)=0 .
$$

Proof. Let $\left(\mu_{n}, n \geq 1\right)$ be a sequence of elements of $\mathcal{M}_{f}(E)$ which converges to $\mu \in \mathcal{M}_{f}(E)$ for the distance $D$ (i.e. which converges weakly). For all $a \geq 0$, we have $\left\langle\left[k_{a} \mu_{n}, \rho\right], 1\right\rangle=$ $\langle\rho, 1\rangle+\left(\left\langle\mu_{n}, 1\right\rangle-a\right)_{+}$and $\lim _{n \rightarrow \infty}\left\langle\left[k_{a} \mu_{n}, \rho\right], 1\right\rangle=\left\langle\left[k_{a} \mu, \rho\right], 1\right\rangle$. There exists a finite constant $A$ such that $\langle\mu, 1\rangle \leq A$ and $\left\langle\mu_{n}, 1\right\rangle \leq A$ for all $n \geq 1$. Furthermore the sequence of functions $\left(G\left(H^{\mu_{n}}\right), n \geq 1\right)$ converges in $L^{1}([0, A], d r)$ to $G\left(H^{\mu}\right)$. Since $G\left(H^{\mu_{n}}\right)$ and $G\left(H^{\mu}\right)$ are nonincreasing, we deduce that $\lim _{n \rightarrow \infty} G\left(H_{a}^{\mu_{n}}\right)=G\left(H_{a}^{\mu}\right)$ for any continuity point $a$ of $G\left(H^{\mu}\right)$. Since $G$ is increasing and continuous, we deduce that for any $a \in \mathcal{A}_{\mu} \cap[0, A]$, we have $\lim _{n \rightarrow \infty} H_{a}^{\mu_{n}}=H_{a}^{\mu}$. The results is also trivially true for $a>A$.

Let $a \in \mathcal{A}_{\mu}$. For $r \leq\langle\rho, 1\rangle$, we have

$$
H_{r}^{\left[k_{a} \mu_{n}, \rho\right]}=H_{a}^{\mu_{n}}+H_{r}^{\rho} \underset{n \rightarrow \infty}{\longrightarrow} H_{a}^{\mu}+H_{r}^{\rho}=H_{r}^{\left[k_{a} \mu, \rho\right]},
$$

and for $r \geq\langle\rho, 1\rangle, d r$-a.e.

$$
H_{r}^{\left[k_{a} \mu_{n}, \rho\right]}=H_{a+r-\langle\rho, 1\rangle}^{\mu_{n}} \underset{n \rightarrow \infty}{\longrightarrow} H_{a+r-\langle\rho, 1\rangle}^{\mu}=H_{r}^{\left[k_{a} \mu, \rho\right]} .
$$

Notice that for $r \geq A+\langle\rho, 1\rangle$, we have $H_{r}^{\left[k_{a} \mu_{n}, \rho\right]}=H_{r}^{\left[k_{a} \mu, \rho\right]}=0$. Since $G$ is continuous, we get by dominated convergence for $a \in \mathcal{A}_{\mu}$, that

$$
\lim _{n \rightarrow \infty} \int_{0}^{\infty} d\left(H_{r}^{\left[k_{a} \mu_{n}, \rho\right]}, H_{r}^{\left[k_{a} \mu, \rho\right]}\right) d r=0 .
$$

This implies that for $a \in \mathcal{A}_{\mu}, \lim _{n \rightarrow \infty} D\left(\left[k_{a} \mu_{n}, \rho\right],\left[k_{a} \mu, \rho\right]\right)=0$.

Let $\mathcal{C}_{0}\left(\mathcal{M}_{f}(E)\right)$ be the set of real continuous functions defined on $\left(\mathcal{M}_{f}(E), D\right)$ such that $\lim _{n \rightarrow \infty} F\left(\mu_{n}\right)=0$ whenever $\lim _{n \rightarrow \infty} D\left(0, \mu_{n}\right)=+\infty$, that is whenever $\lim _{n \rightarrow \infty}\left\langle\mu_{n}, 1\right\rangle=\infty$.

Corollary 3.6. Let $\left(I^{*}, \rho\right)$ be a random variable with values in $\mathbb{R}_{+}, \times \mathcal{M}_{f}(E)$, such that the distribution of $I^{*}$ has no atom (i.e. $\mathbb{P}\left(I^{*}=x\right)=0$ for all $\left.x \in \mathbb{R}_{+}\right)$. Let $F \in \mathcal{C}_{0}\left(\mathcal{M}_{f}(E)\right)$. Then the function $\mathcal{F}: \mu \mapsto \mathbb{E}\left[F\left(\left[k_{I^{*}} \mu, \rho\right]\right)\right]$ belongs to $\mathcal{C}_{0}\left(\mathcal{M}_{f}(E)\right)$.

Proof. Let $Q$ be the distribution of $I^{*}$ and $\left(P_{a}(d \rho), a \geq 0\right)$ be a measurable version of the conditional law of $\rho$ knowing $I^{*}: P_{a}(d \rho) Q(d a)$ is the distribution of $\left(I^{*}, \rho\right)$. For $F \in \mathcal{C}_{0}\left(\mathcal{M}_{f}(E)\right)$, we have

$$
\mathbb{E}\left[F\left(\left[k_{I^{*}} \mu, \rho\right]\right)\right]=\int P_{a}\left[F\left(\left[k_{a} \mu, \rho\right]\right)\right] Q(d a) .
$$

Let $\left(\mu_{n}, n \leq 1\right)$ be a sequence converging to $\mu$. From Lemma 3.5. for all $a \in \mathcal{A}_{\mu}$, $\lim _{n \rightarrow \infty} D\left(\left[k_{a} \mu_{n}, \rho\right],\left[k_{a} \mu, \rho\right]\right)=0$. Since the complementary of $\mathcal{A}_{\mu}$ is at most countable and 
since $Q(d a)$ has no atoms, we get that $Q(d a)$-a.s. $\lim _{n \rightarrow \infty} F\left(\left[k_{a} \mu_{n}, \rho\right]\right)=F\left(\left[k_{a} \mu, \rho\right]\right)$, for any $\rho \in$ $\mathcal{M}_{f}(E)$. Notice $F$ is bounded. By dominated convergence, we get that $\lim _{n \rightarrow \infty} \mathbb{E}\left[F\left(\left[k_{I^{*}} \mu_{n}, \rho\right]\right)\right]=$ $\mathbb{E}\left[F\left(\left[k_{I^{*}} \mu, \rho\right]\right)\right]$. Thus the function $\mathcal{F}$ is continuous.

Notice that the total mass of $\left[k_{I^{*}} \mu, \rho\right]$ is equal to $\left(\langle\mu, 1\rangle-I^{*}\right)_{+}+\langle\rho, 1\rangle$. If $\lim _{n \rightarrow \infty}\left\langle\mu_{n}, 1\right\rangle=\infty$, then a.s. we have $\lim _{n \rightarrow \infty} F\left(\left[k_{I^{*}} \mu_{n}, \rho\right]\right)=0$. By the dominated convergence Theorem, we get $\lim _{n \rightarrow \infty} \mathcal{F}\left(\mu_{n}\right)=0$.

In conclusion, we get that $\mathcal{F} \in C_{0}\left(\mathcal{M}_{f}(E)\right)$.

3.2. Feller property: Proof of Theorem 2.1. Let $\left(P_{t}, t \geq 0\right)$ be the transition semi-group of the Markov process $\rho$ on $\mathcal{M}_{f}(E)$.

Recall $\mathcal{C}_{0}\left(\mathcal{M}_{f}(E)\right)$ is the set of real continuous functions defined on $\left(\mathcal{M}_{f}(E), D\right)$ such that $\lim _{n \rightarrow \infty} F\left(\mu_{n}\right)=0$ whenever $\lim _{n \rightarrow \infty} D\left(0, \mu_{n}\right)=+\infty$, that is whenever $\lim _{n \rightarrow \infty}\left\langle\mu_{n}, 1\right\rangle=\infty$.

Let us recall that (see e.g. [8], Proposition III.2.4) $\rho$ is a Feller process if and only if

(i) If $F \in \mathcal{C}_{0}\left(\mathcal{M}_{f}(E)\right)$, then for every $t \geq 0, P_{t} F \in \mathcal{C}_{0}\left(\mathcal{M}_{f}(E)\right)$.

(ii) For every $F \in \mathcal{C}_{0}\left(\mathcal{M}_{f}(E)\right)$, for every $\mu \in \mathcal{M}_{f}(E), \lim _{t \rightarrow 0} P_{t} F(\mu)=F(\mu)$.

To prove Condition (i), we state the next Lemma.

Lemma 3.7. For every $t>0$, the distribution of $I_{t}$ has no atom.

Proof. Let us denote by $\tau=\left(\tau_{r}, r \geq 0\right)$ the right-continuous inverse of the process $\left(-I_{t}, t \geq 0\right)$. We now (cf 3], Chap. VII) that the process $\tau$ is a subordinator with Laplace exponent $\psi^{-1}$. As $\lim _{\lambda \rightarrow \infty} \psi(\lambda) / \lambda=\infty$, we have that $\lim _{\lambda \rightarrow \infty} \psi^{-1}(\lambda) / \lambda=0$. The process $\tau$ has no drift. It is moreover strictly increasing since the process $-I_{t}$ is continuous. It is easy to check that if $\mathbb{P}\left(-I_{t}=x\right)>0$ for some $x>0$, then we have $\mathbb{P}\left(-I_{t}=x, \tau_{x}=t\right)>0$ and $\mathbb{P}\left(\tau_{-I_{t}}=t\right)>0$. This is in contradiction to the fact that $\tau$ has no drift (see Theorem 4 in [3]). Hence the Lemma is proved.

Let $F \in \mathcal{C}_{0}\left(\mathcal{M}_{f}(E)\right)$ and let $t \geq 0$. Then, as the distribution of the random variable $I_{t}$ has no atoms (see Lemma [3.7), we can apply Corollary [3.6 with $I^{*}=-I_{t}$ and get the function $\mathcal{F}: \mu \mapsto \mathbb{E}_{\mu}\left[F\left(\rho_{t}^{\mu}\right)\right]$ belongs to $\mathcal{C}_{0}\left(\mathcal{M}_{f}(E)\right)$.

It remains to prove Condition (ii). Let us remark that the exploration process is rightcontinuous at $t=0$. Indeed, for every continuous function $f$ on $E$ bounded by 1 , we have, $\mathbb{P}_{\mu}$-a.s.

$$
\begin{aligned}
\left|\left\langle\rho_{t}, f\right\rangle-\langle\mu, f\rangle\right| & =\left|\left\langle\left[k_{-I_{t}} \mu, \rho_{t}^{0}\right], f\right\rangle-\langle\mu, f\rangle\right| \\
& =\left|-\left\langle\mu-k_{-I_{t}} \mu, f\right\rangle+\left\langle\rho_{t}^{0}, f\left(H_{-I_{t}}^{\mu}+\cdot\right)\right\rangle\right| \\
& \leq\left\langle\mu-k_{-I_{t}} \mu, 1\right\rangle+\left\langle\rho_{t}^{0}, 1\right\rangle \\
& \leq-I_{t}+X_{t}-I_{t} .
\end{aligned}
$$

And the right continuity of $\rho$ at 0 follows from the right-continuity of $X$ and $I$ at 0 . Let $F \in \mathcal{C}_{0}\left(\mathcal{M}_{f}(E)\right)$. As the function $F$ is bounded, we get by dominated convergence that, for every $\mu \in \mathcal{M}_{f}(E)$,

$$
\mathbb{E}_{\mu}\left[F\left(\rho_{t}\right)\right] \underset{t \rightarrow 0}{\longrightarrow} F(\mu)
$$

\section{Proof of Theorem 2.2}

4.1. Other properties of the exploration process. In this section we recall some properties of the exploration process. 
4.1.1. Poisson decomposition. Let $\mu \in \mathcal{M}_{f}(E)$. We decompose the path of $\rho$ under $\mathbb{P}_{\mu}$ according to excursions of the total mass of $\rho$ above its minimum. More precisely, we denote by $\left(\alpha_{i}, \beta_{i}\right), i \in \mathcal{I}$ the excursion intervals of $X-I$ away from 0 . For every $i \in \mathcal{I}, t \in\left(\alpha_{i}, \beta_{i}\right)$, we have $\rho_{\alpha_{i}}=k_{-I_{\alpha_{i}}} \mu=k_{-I_{\alpha_{i}}} \rho_{t}$. We define $\rho^{i}$ by the formula $\rho_{t}^{i}=\rho_{\left(\alpha_{i}+t\right) \wedge \beta_{i}}^{0}$ or equivalently if $\mu \in \mathcal{M}_{f}$ :

$$
\left[k_{-I_{\alpha_{i}}} \mu, \rho_{t}^{i}\right]=\rho_{\left(\alpha_{i}+t\right) \wedge \beta_{i}} .
$$

The local time of $X-I$ at level 0 is given by $-I$, and if $\left(\tau_{r}, r \geq 0\right)$ is the right continuous inverse of $I$, then the process $\left(\tau_{r}, r \geq 0\right.$ ) is a subordinator with Laplace exponent $\psi^{-1}$ (cf [3] Chap. VII). Excursion theory for $X-I$ ensures the following result.

Lemma 4.1. Let $\mu \in \mathcal{M}_{f}(E)$. The point measure $\sum_{i \in I} \delta_{\left(-I_{\alpha_{i}}, \rho^{i}\right)}$ is under $\mathbb{P}_{\mu}$ a Poisson point measure with intensity $d r \mathbb{N}[d \rho]$.

Let $F$ be a non-negative measurable function defined on $\mathbb{R}_{+} \times \mathcal{M}_{f}(E) \times \mathbb{D}\left([0, \infty), \mathcal{M}_{f}(E)\right)$, where $\mathbb{D}\left([0, \infty), \mathcal{M}_{f}(E)\right)$ stands for the Skorohod space of càd-làg path in $\mathcal{M}_{f}(E)$. We have

$$
\mathbb{E}_{\mu}\left[\sum_{i \in \mathcal{I}} F\left(\alpha_{i}, \rho_{\alpha_{i}}, \rho^{i}\right)\right]=\mathbb{E}_{\mu}\left[\int_{0}^{\infty} d r \mathbb{N}\left[F\left(v, k_{r} \mu, \rho\right)\right]_{\mid v=\tau_{r}}\right] .
$$

4.1.2. The dual process and representation formula. We shall need the $\mathcal{M}_{f}$-valued process $\eta=\left(\eta_{t}, t \geq 0\right)$ defined under $\mathbb{N}$ by

$$
\eta_{t}(d r)=\sum_{\substack{0<s \leq t \\ X_{s-}<I_{t}^{s}}}\left(X_{s}-I_{t}^{s}\right) \delta_{H_{s}}(d r) .
$$

The process $\eta$ is the dual process of $\rho$ under $\mathbb{N}$ (see Corollary 3.1.6 in [4]). Let $\sigma=\inf \{s>$ $\left.0 ; \rho_{s}=0\right\}$ denote the length of the excursion. Using this duality, it easy to check (see the proof of Lemma 3.2.2 in [4]), that for any bounded measurable function $F$ defined on $\mathcal{M}_{f}$,

$$
\mathbb{N}\left[\int_{0}^{\sigma} \mathrm{e}^{-\psi(\gamma) t} F\left(\rho_{t}\right)\right]=\mathbb{N}\left[\int_{0}^{\sigma} \mathrm{e}^{-\gamma\left\langle\eta_{t}, 1\right\rangle} F\left(\rho_{t}\right)\right] .
$$

We recall the Poisson representation of $(\rho, \eta)$ under $\mathbb{N}$. Let $\mathcal{N}(d x d \ell d u)$ be a Poisson point measure on $[0,+\infty)^{3}$ with intensity

$$
d x \ell \pi(d \ell) \mathbf{1}_{[0,1]}(u) d u .
$$

For every $a>0$, let us denote by $\mathbb{M}_{a}$ the law of the pair $\left(\mu_{a}, \nu_{a}\right)$ of finite measures on $\mathbb{R}_{+}$ defined by: for $f \in \mathcal{B}_{+}\left(\mathbb{R}_{+}\right)$

$$
\begin{aligned}
\left\langle\mu_{a}, f\right\rangle & =\int \mathcal{N}(d x d \ell d u) \mathbf{1}_{[0, a]}(x) u \ell f(x), \\
\left\langle\nu_{a}, f\right\rangle & =\int \mathcal{N}(d x d \ell d u) \mathbf{1}_{[0, a]}(x) \ell(1-u) f(x) .
\end{aligned}
$$

We eventually set $\mathbb{M}=\int_{0}^{+\infty} d a \mathrm{e}^{-\alpha_{0} a} \mathbb{M}_{a}$.

Proposition 4.2. For every non-negative measurable function $F$ on $\mathcal{M}_{f}^{2}$,

$$
\mathbb{N}\left[\int_{0}^{\sigma} F\left(\rho_{t}, \eta_{t}\right) d t\right]=\int \mathbb{M}(d \mu d \nu) F(\mu, \nu)
$$


4.2. Computation of the resolvent. Let $f$ be a bounded function defined on $\mathbb{R}_{+}$of class $\mathcal{C}^{1}$ with bounded first derivative such that $f(\infty)=\lim _{t \rightarrow \infty} f(t)$ exists. By convention, we put $f^{\prime}(\infty)=0$.

We set for $\mu \in \mathcal{M}_{f}(E)$

$$
F_{\lambda}(\mu)=\mathrm{e}^{-\langle\mu, f\rangle}\left(\lambda-\psi\left(f\left(H^{\mu}\right)\right)+f^{\prime}\left(H^{\mu}\right)\right) .
$$

Let $\gamma=\psi^{-1}(\lambda)$. We shall compute

$$
U_{\lambda}\left(F_{\lambda}\right)(\mu)=\mathbb{E}_{\mu}\left[\int_{0}^{\infty} d t \mathrm{e}^{-\psi(\gamma) t-\left\langle\rho_{t}, f\right\rangle}\left(\psi(\gamma)-\psi\left(f\left(H_{t}\right)\right)+f^{\prime}\left(H_{t}\right)\right)\right] .
$$

We define the function $f_{r}$ by $f_{r}(\cdot)=f\left(\cdot+H_{r}^{\mu}\right)$, where $H_{r}^{\mu}=H^{k_{r} \mu}$ and use the convention $x+\infty=x$ for all $x \in E$. Using notations of Section 4.1.1 and (9), we have

$$
\begin{aligned}
U_{\lambda}\left(F_{\lambda}\right)(\mu)= & \mathbb{E}_{\mu}\left[\sum_{i \in \mathcal{I}} \mathrm{e}^{-\psi(\gamma) \alpha_{i}} \int_{0}^{\beta_{i}-\alpha_{i}} d t \mathrm{e}^{-\psi(\gamma) t-\left\langle\left[k_{-I_{\alpha_{i}}} \mu, \rho_{t}^{i}\right], f\right\rangle}\right. \\
& \left.\left(\psi(\gamma)-\psi\left(f_{-I_{\alpha_{i}}}\left(H^{\rho_{t}^{i}}\right)\right)+f_{-I_{\alpha_{i}}}^{\prime}\left(H^{\rho_{t}^{i}}\right)\right)\right] \\
= & \mathbb{E}_{\mu}\left[\int_{0}^{\infty} d r \mathrm{e}^{-\psi(\gamma) \tau_{r}} \mathbb{N}\left[\int_{0}^{\sigma} d t \mathrm{e}^{-\psi(\gamma) t-\left\langle\left[k_{r} \mu, \rho_{t}\right], f\right\rangle}\left(\psi(\gamma)-\psi\left(f_{r}\left(H_{t}\right)\right)+f_{r}^{\prime}\left(H_{t}\right)\right)\right]\right] .
\end{aligned}
$$

Recall $\left(\tau_{r}, r \geq 0\right)$ is a subordinator with Laplace exponent $\psi^{-1}$ and (10). Since $\left\langle\left[k_{r} \mu, \rho_{t}\right], f\right\rangle=$ $\left\langle k_{r} \mu, f\right\rangle+\left\langle\rho_{t}, f_{r}\right\rangle$, we get

$$
U_{\lambda}\left(F_{\lambda}\right)(\mu)=\int_{0}^{\infty} d r \mathrm{e}^{-\gamma r} \mathrm{e}^{-\left\langle k_{r} \mu, f\right\rangle} \mathbb{N}\left[\int_{0}^{\sigma} d t \mathrm{e}^{-\gamma\left\langle\eta_{t}, 1\right\rangle-\left\langle\rho_{t}, f_{r}\right\rangle}\left(\psi(\gamma)-\psi\left(f_{r}\left(H_{t}\right)\right)+f_{r}^{\prime}\left(H_{t}\right)\right)\right] .
$$

We deduce from Proposition 4.2 that

$$
\begin{gathered}
\mathbb{N}\left[\int_{0}^{\sigma} d t \mathrm{e}^{-\gamma\left\langle\eta_{t}, 1\right\rangle-\left\langle\rho_{t}, f_{r}\right\rangle}\left(\psi(\gamma)-\psi\left(f_{r}\left(H_{t}\right)\right)+f_{r}^{\prime}\left(H_{t}\right)\right)\right] \\
=\int_{0}^{\infty} d a \mathrm{e}^{-\alpha_{0} a}\left(\psi(\gamma)-\psi\left(f_{r}(a)\right)+f_{r}^{\prime}(a)\right) \\
\quad \exp \left\{-\int_{0}^{a} d x \int_{0}^{1} d u \int_{(0, \infty)} \ell \pi(d \ell)\left[1-\mathrm{e}^{-\gamma(1-u) \ell-u f_{r}(x) \ell}\right]\right\} \\
=\int_{0}^{\infty} d a\left(\psi(\gamma)-\psi\left(f\left(a+H_{r}^{\mu}\right)\right)+f^{\prime}\left(a+H_{r}^{\mu}\right)\right) \mathrm{e}^{-\int_{0}^{a} d x \int_{0}^{1} d u \psi^{\prime}\left(\gamma(1-u)+u f\left(x+H_{r}^{\mu}\right)\right)} .
\end{gathered}
$$

We set for $y \in E$

$$
\Lambda(y)=\int_{0}^{\infty} d a\left(\psi(\gamma)-\psi(f(a+y))+f^{\prime}(a+y)\right) \mathrm{e}^{-g(a, y)},
$$

where $g(a, y)=\int_{0}^{a} d x \frac{\psi(f(x+y))-\psi(\gamma)}{f(x+y)-\gamma}$ with the convention $\frac{\psi(v)-\psi(v)}{v-v}=\psi^{\prime}(v)$, so that

$$
U_{\lambda}\left(F_{\lambda}\right)(\mu)=\int_{0}^{\infty} d r \mathrm{e}^{-\gamma r} \mathrm{e}^{-\left\langle k_{r} \mu, f\right\rangle} \Lambda\left(H_{r}^{\mu}\right)
$$


Since $\psi$ is positive increasing continuous and $f$ bounded, we have $\lim _{a \rightarrow \infty} g(a, y)=\infty$. Notice that for $y \in[0, \infty)$

$$
\begin{aligned}
\Lambda(y) & =\int_{0}^{\infty} d a\left(-\partial_{a} g(a, y)(f(a+y)-\gamma)+f^{\prime}(a+y)\right) \mathrm{e}^{-g(a, y)} \\
& =\left[(f(a+y)-\gamma) \mathrm{e}^{-g(a, y)}\right]_{0}^{\infty} \\
& =\gamma-f(y) .
\end{aligned}
$$

We also have $\Lambda(\infty)=\gamma-f(\infty)$. We deduce from (14) that

$$
U_{\lambda}\left(F_{\lambda}\right)(\mu)=\int_{0}^{\infty} d r \mathrm{e}^{-\gamma r-\left\langle k_{r} \mu, f\right\rangle}\left(\gamma-f\left(H_{r}^{\mu}\right)\right)
$$

Notice that $\left\langle k_{r} \mu, f\right\rangle=0$ and $H_{r}^{\mu}=0$ for $\left.r\right\rangle\langle\mu, 1\rangle$, so that we have

$$
\int_{\langle\mu, 1\rangle}^{\infty} d r \mathrm{e}^{-\gamma r-\left\langle k_{r} \mu, f\right\rangle}\left(\gamma-f\left(H_{r}^{\mu}\right)\right)=\int_{\langle\mu, 1\rangle}^{\infty} d r \mathrm{e}^{-\gamma r}(\gamma-f(0))=\mathrm{e}^{-\gamma\langle\mu, 1\rangle}\left(1-\frac{f(0)}{\gamma}\right) .
$$

We deduce from (6) with $\mu$ replaced by $k_{r} \mu$ that for $r \leq\langle\mu, 1\rangle$,

$$
\left\langle k_{r} \mu, f\right\rangle=\int_{0}^{\langle\mu, 1\rangle-r} f\left(H_{v}^{k_{r} \mu}\right) d v=\int_{r}^{\langle\mu, 1\rangle} f\left(H_{v}^{\mu}\right) d v .
$$

This implies that

$$
\begin{aligned}
\int_{0}^{\langle\mu, 1\rangle} d r \mathrm{e}^{-\gamma r-\left\langle k_{r} \mu, f\right\rangle}\left(\gamma-f\left(H_{r}^{\mu}\right)\right) & =\int_{0}^{\langle\mu, 1\rangle} d r \mathrm{e}^{-\gamma r-\int_{r}^{\langle\mu, 1\rangle} f\left(H_{v}^{\mu}\right) d v}\left(\gamma-f\left(H_{r}^{\mu}\right)\right) \\
& =\left[-\mathrm{e}^{-\gamma r-\int_{r}^{\langle\mu, 1\rangle} f\left(H_{v}^{\mu}\right) d v}\right]_{0}^{\langle\mu, 1\rangle} \\
& =\mathrm{e}^{-\langle\mu, f\rangle}-\mathrm{e}^{-\gamma\langle\mu, 1\rangle},
\end{aligned}
$$

where we used (6) for the first term of the right hand side of the last equation. Eventually, we have

$$
U_{\lambda}\left(F_{\lambda}\right)(\mu)=\mathrm{e}^{-\langle\mu, f\rangle}-\frac{f(0)}{\gamma} \mathrm{e}^{-\gamma\langle\mu, 1\rangle}
$$

\section{REFERENCES}

[1] R. ABRAHAM and J.-F. DELMAS. Fragmentation associated to Lévy processes. Preprint CERMICS, 2005.

[2] D. ALDOUS. The continuum random tree III. Ann. Probab., 21(1):248-289, 1993.

[3] J. BERTOIN. Lévy processes. Cambridge University Press, Cambridge, 1996.

[4] T. DUQUESNE and J.-F. LE GALL. Random trees, Lévy processes and spatial branching processes, volume 281. Astérisque, 2002.

[5] S. N. ETHIER and T. G. KURTZ. Markov processes. Wiley, 1986.

[6] J.-F. LE GALL and Y. LE JAN. Branching processes in Lévy processes: Laplace functionals of snake and superprocesses. Ann. Probab., 26:1407-1432, 1998.

[7] J.-F. LE GALL and Y. LE JAN. Branching processes in Lévy processes: The exploration process. Ann. Probab., 26:213-252, 1998.

[8] D. REVUZ and M. YOR. Continuous martingales and Brownian motion. Springer Verlag, 2nd edition, 1995. 
Romain Abraham, MAPMO, Université d'Orléans, B.P. 6759, 45067 Orléans Cedex 2 France

E-mail address: romain.abraham@univ-orleans.fr

Jean-François Delmas, EnPC-CERMiCS, 6-8 av. Blaise Pascal, Champs-sur-Marne, 77455 Marne la Vallée, France.

E-mail address: delmas@cermics.enpc.fr 